\title{
Lipid Peroxidation and Polybrominated Diphenyl Ethers - A Toxicological Perspective
}

\author{
Mary C. Vagula and Elisa M. Konieczko \\ Additional information is available at the end of the chapter
}

http://dx.doi.org/10.5772/50345

\section{Introduction}

Understanding the structure and composition of plasma membrane is important as it guards the integrity and function of the cell as a whole. Lipid peroxidation is a process in which the lipids of the cell, particularly the membrane lipids, are degraded by oxidation resulting in the disruption of the entire cell. All cells are enclosed by a plasma membrane which serves as a boundary and it is the primary barrier between cell's internal and external environments. It is semi-permeable and plays a major role in regulating the transport of molecules into and out of the cells. This property of the plasma membrane is known as selective permeability. The fluid mosaic model describes the arrangement of the lipids and the proteins in the plasma membrane [1]. The lipids are smaller in size than the proteins. However, there are many more lipids than there are proteins. The lipids give the membranes their basic shape. Membrane lipids are amphipathic molecules, with hydrophilic and hydrophobic ends [2]. These lipids arrange in two layers (bilayer) such that the hydrophobic ends of one layer touches the hydrophobic ends of the other lipid layer. The hydrophilic ends of the phospholipids are in contact with the aqueous external and internal environments. Membrane lipids include phospholipids, cholesterol, sphingomyelin, triacylglycerides and glycolipids [3]. Several different types of phospholipids are found in cellular membranes: phosphatidylcholine (the most abundant), phosphatidylserine, phosphatidylinositol and phosphatidylethanolamime [4]. Each species of organisms has its own unique combination of these different phospholipids in its cellular membranes.

The proteins of the membrane float in the lipid bilayer. There are fewer proteins than lipids in the plasma membrane. However, they are much bigger than the lipids. The proteins can be associated with the lipids of the bilayer in one of the three ways [5]. Integral proteins are amphipathic molecules that span the entire width of lipid bilayer. Their hydrophilic and hydrophobic portions allow them to interact with both lipids and the aqueous environments 
on either side of the lipids. Membrane associated proteins are associated with either external or internal surfaces of the membrane. These proteins often contain a lipid anchor which helps to hold them in the membrane. Peripheral proteins are attached to the inner surface of the membrane through covalent bonds to another protein. For example, the proteins that link the plasma membrane to the cytoskeleton. Plasma membrane proteins have several functions [6]. They serve as transporters, receptors, enzymes and as anchors, tying the cell to the external environment or to other cells. Carbohydrates attached to some proteins serve as identification markers and help in the differentiation of self from non-self cells [7].

The composition and structure of the plasma membrane described above is affected by free radicals. So the next section deals with free radicals, on how they are generated in the cells and their effects on cellular macromolecules.

\section{Free radicals and Reactive Oxygen Species (ROS)}

A free radical may be defined as any atom or molecule that has one or more unpaired electrons in its outermost shell. Free radicals were discovered in biological material about 50 years ago [8] and since then, they have generated great curiosity for their involvement in disease and aging. Oxygen free radicals or reactive oxygen species (ROS) are defined as any free radical molecules containing oxygen. Some examples of the most common ROS include: hydrogen peroxide $\left(\mathrm{H}_{2} \mathrm{O}_{2}\right)$, hydroxyl $\left({ }^{\bullet} \mathrm{OH}\right)$, and superoxide $\left(\mathrm{O}_{2}{ }^{\bullet}-\right)$ anion. Under normal conditions, ROS are produced as necessary intermediates in white blood cells to defend against invading pathogens, and they also play a role in intracellular signaling. Owing to their unpaired electrons in their outermost shell, ROS are highly reactive and unstable and they exert damage on biological structures. Free radicals react quickly with other molecules and "steal" electrons to acquire stability. The molecule which lost its electron to a free radical will become a free radical itself, thus beginning a chain reaction. Free radicals modify the structure of other molecules and cause oxidative damage. Once the process is started, if not quenched in time, it can eventually result in cell damage. Additionally, excessive amounts of free radicals are generated when tissues are damaged, for example, in times of tissue hypoxia, exposure to smoke, ultraviolet radiation and pollutants.

\subsection{Generation of reactive oxygen species}

Most of the energy required to fuel metabolic functions of aerobic organisms is produced at the cellular level in the mitochondria via the electron transport chain. In addition to energy, reactive oxygen species (ROS) are also generated from electron transport chain as part of normal cellular metabolic reactions. In this transport chain, ROS are formed at the mitochondrial inner membrane. Under normal conditions, the oxygen molecule acts as a terminal electron acceptor in the electron transport chain and is reduced to form water. However, sometimes electrons leak prematurely and reduce oxygen to form ROS. The electron transfer between ubiquinone-cytochrome $b$ is the most probable site of ROS formation [9]. Microsomes and membranes of the nucleus also involve electron transportation through the cytochromes $\mathrm{P}_{450}$ and $\mathrm{B}_{5}$ which can produce free radicals [10]. The following few paragraphs discuss the generation of various ROS. 


\subsection{Superoxide anion $\left(\mathrm{O}_{2} \cdot-\right)$}

When ground state oxygen accepts a single electron a superoxide anion $\left(\mathrm{O}_{2}{ }^{--}\right)$is formed.

$$
\mathrm{O}_{2}+\mathrm{e}^{-} \rightarrow \mathrm{O}_{2} \cdot-
$$

Under normal physiological conditions cell organelles, namely, mitochondria, microsomes and peroxisomes, generate $\mathrm{O}_{2}{ }^{\bullet}$. The half-life of superoxide anion is longer than that of another potent ROS, hydroxyl radical $\left(\mathrm{OH}^{*}\right)$, and it can react with biological molecules for longer times [10]. Phagocytic cells produce superoxide during a phenomenon known as the "respiratory burst" which occurs when they encounter pathogens. Phagocytic cells known to produce superoxide anions are macrophages, monocytes, neutrophils and eosinophils [11]. Superoxide anions can trigger the formation of hydroxyl and peroxyl radicals, which in an acidic environment can form hydrogen peroxide $\left(\mathrm{H}_{2} \mathrm{O}_{2}\right)$.

\subsection{Hydrogen peroxide $\left(\mathrm{H}_{2} \mathrm{O}_{2}\right)$}

The addition of a second electron to $\mathrm{O}_{2}{ }^{-}$- gives rise to the peroxide ion, $\mathrm{O}_{2}{ }^{2-}$. At physiological $\mathrm{pH}$ any peroxide ion formed is protonated to hydrogen peroxide $\left(\mathrm{H}_{2} \mathrm{O}_{2}\right)$.

$$
2 \mathrm{O}_{2} \cdot \cdot+2 \mathrm{H}^{+} \rightarrow \mathrm{H}_{2} \mathrm{O}_{2}+\mathrm{O}_{2}
$$

Hydrogen peroxide can then diffuse far from the site of its production to other sites where its biological effects may be greater. The diffusion range is extended by $\mathrm{H}_{2} \mathrm{O}_{2}$ carriers formed spontaneously by hydrogen bonding with compounds such as amino and dicarboxylic acids, peptides, proteins, nucleic acid bases, and nucleosides. Equilibrium exists between an adduct-forming compound and $\mathrm{H}_{2} \mathrm{O}_{2}$. The hydrogen peroxide adducts (HPAs) retard the decomposition of $\mathrm{H}_{2} \mathrm{O}_{2}$ up to several hundredfold [12]. The overall charge on an HPA influences the cytotoxic and clastogenic effects of $\mathrm{H}_{2} \mathrm{O}_{2}$. The adducts, especially L- Histidine, play an important role in the stabilization and reduction in the reactivity of the hydrogen peroxide thereby preventing single strand breaks of the DNA in cell free DNA systems [13].

\subsection{Hydroxyl radical $(\cdot \mathrm{OH})$}

The hydroxyl radical $\left({ }^{\bullet} \mathrm{OH}\right)$ can be formed by the homolytic fission of the $\mathrm{O}-\mathrm{O}$ bond of the $\mathrm{H}_{2} \mathrm{O}_{2}$ molecule. Simple mixing of iron (II) salts with the $\mathrm{H}_{2} \mathrm{O}_{2}$ also forms the ${ }^{\bullet} \mathrm{OH}$ radical. This reaction was first reported by Fenton in 1894 and it is called Fenton's reaction. The hydroxyl radicals can also be produced by Haber-weiss reactions in the cells, in which $\mathrm{H}_{2} \mathrm{O}_{2}$ interacts with superoxide radical [10]. Additionally, copper salts can also react with $\mathrm{H}_{2} \mathrm{O}_{2}$ to generate ${ }^{\bullet} \mathrm{OH}$ radicals. All the three ways of ${ }^{\bullet} \mathrm{OH}$ radical generation is shown below:

By the decomposition of $\mathrm{H}_{2} \mathrm{O}_{2}$ by Fenton's reaction:

$$
\mathrm{Fe}^{+2}+\mathrm{H}_{2} \mathrm{O}_{2} \rightarrow \mathrm{Fe}^{+3}+\mathrm{OH}+\mathrm{OH}^{-}
$$

and $\mathrm{H}_{2} \mathrm{O}_{2}$ interaction with superoxide radical by the Haber-Weiss reaction: 


$$
\mathrm{O}_{2}--\mathrm{H}_{2} \mathrm{O}_{2} \rightarrow \mathrm{O}_{2}+\mathrm{H}_{2} \mathrm{O}+\mathrm{OH}
$$

The hydroxyl radical can also be formed when copper salts react with $\mathrm{H}_{2} \mathrm{O}_{2}$ to make hydroxyl radicals as shown below:

$$
\mathrm{Cu}^{+}+\mathrm{H}_{2} \mathrm{O}_{2} \rightarrow \mathrm{Cu}^{2+}+\cdot \mathrm{OH}+\mathrm{OH}^{-}
$$

Although the hydroxyl radical $(\mathrm{OH})$ has the shortest half-life, it is one of the most reactive of all ROS [14, 15]. Due to this property it reacts with almost all macromolecules found in the cells such as proteins, nucleic acids (RNA and DNA), phospholipids, sugars and carbohydrates, and exerts deleterious effects on them.

\subsection{Free radicals and the damage to cellular components}

Excess generation of free radicals combined with the failure of cells to scavenge them through protective mechanisms (antioxidative agents and enzymes) results in oxidative damage. Oxidative damage is witnessed in the form of free radical mediated attack on macromolecules within their vicinity and causing disruption of these molecules. Severe oxidative stress can even result in apoptosis and cell death [16]. Free radical mediated damage to proteins, carbohydrates, nucleic acids and lipids is described below.

\subsection{Free radical damage to proteins}

The oxidization reaction of free radicals on amino acids causes changes in the physical properties of proteins that they compose. These physical changes are of three types: fragmentation, aggregation and susceptibility to enzyme digestion [17]. The fragmentation of albumin and collagen due to free radical mediated oxidization is a classic example of this phenomenon $[18,19]$. Selective fragmentation at specific amino acids, especially at proline, histidine and arginine, is observed due to their close association with transition metals. The second type of physical change, protein aggregation, is believed to be caused predominantly by hydroxyl radicals due to their ability to form cross-links between the constitutive amino acids. Lastly, gross alteration in the conformation of protein structure may be the reason for the susceptibility of these altered proteins to enzyme digestion [10].

\subsection{Free radical damage to carbohydrates}

Hydroxyl radicals generated by Fenton reaction are reported to induce damage on simple carbohydrates [20]. It is also reported that oxidation of glucose may be a means of quenching hydroperoxide radicals while also generating a source of oxygen free radicals [21]. Another study indicated that rapid auto-oxidation of glucose results in the formation of dicarbonyl and $\mathrm{H}_{2} \mathrm{O}_{2}$ under physiological conditions [22]. Thus, oxidized glucose can react with proteins in a process called glucosylation or glucation leading to the disruption in the function of these macromolecules. Glucosylation of certain long-lived proteins by a nonenzymic reaction with free glucose may contribute to ageing. 


\subsection{Free radicals and the alteration of gene expression}

Free radicals are extensively implicated for their role in DNA damage and alteration in the gene expression leading to various types of cancers [23]. It has been reported that about twenty types of changes are induced by ROS in the DNA. The damage in the DNA's deoxyguanosine residues is estimated to be in the range of 8 to 83 residues per million deoxyguanosine residues. This damage increases with age and seems to affect organs such as the liver, kidney and spleen while leaving brain tissue relatively undamaged [24]. Mitochondrial DNA experiences greater damage due to proximity to the ROS generated from the electron transport chain of mitochondrial membranes. Free radical induced damage to DNA includes a range of specifically oxidized purines and pyrimidines, alkali labile sites, single strand breaks and instability formed directly or by repair processes $[25,26]$. The pyrimidine residues, cytosine and thymine, of DNA are the most susceptible to the attack by hydroxyl radical, followed in decreasing levels of susceptibility by adenine, guanine and deoxyribose sugar [10]. Some of these modified bases have been found to possess mutagenic properties. Therefore, if not repaired they can lead to carcinogenesis.

\subsection{Free radicals and lipid peroxidation}

Free radicals steal electrons from the cell membrane's polyunsaturated fatty acids (PUFAs) and initiate an attack called lipid peroxidation. The peroxidation of lipids occurs in three steps, namely, initiation, propagation and termination. Free radicals target the carboncarbon double bond of PUFAs, as this double-bond weakens the carbon-hydrogen bond, permitting easy dissociation of the hydrogen by a free radical. During the first step, a single electron is stripped by a free radical from hydrogen atoms associated with the carbon at the double-bond leaving the carbon with a single electron. Molecular arrangements occur in an attempt to stabilize, leading to the formation of conjugated dienes. In the propagation phase, the conjugated diene readily reacts with molecular oxygen and transitions to a hydroperoxide radical that begins stealing electrons from other lipid molecules, resulting in a lipid hydroperoxide [11]. The hydroperoxide molecules undergo decomposition with the aid of transition metals into alcoxyl and peroxyl radicals that, in turn, can initiate a chain reaction and further propagate lipid peroxidation and cause damage to the cell. The three phases or steps of lipid peroxidation are shown in the following equations:

Initiation reaction

Lipid $-\mathrm{H}+\mathrm{OH} \rightarrow$ Lipid $+\mathrm{H}_{2} \mathrm{O}$

Hydroperoxide formation

Lipid $+\mathrm{O}_{2} \rightarrow$ Lipid- $\mathrm{O}_{2}$

Lipid hydroperoxide

Lipid- $\mathrm{O}_{2}+$ Lipid $-\mathrm{H} \rightarrow$ Lipid + Lipid $-\mathrm{O}_{2} \mathrm{H}$

The important fatty acids that undergo peroxidation are: linoleic acid, arachidonic acid, and docosahexaenoic acids [14]. The hydroxyl radical damages cell membranes and other 
lipoproteins by lipid peroxidation. It is important to note that the low density lipoprotein based lipid peroxidation plays a significant role in atherosclerosis [27].

The generation of free radicals and their action on macromolecules thus leads to disruption of cellular functions. The following section discusses chemicals that are present in the body to protect against these damaging radicals.

\section{Antioxidative protection against reactive oxygen species (Free radicals)}

All aerobic cells have an armory of chemicals known as antioxidants which are capable of counteracting the damaging effects of oxidative free radicals and inhibiting the oxidation of other molecules in the body. Based on the mechanism of their action, these antioxidants are divided into two categories: chain breaking antioxidants and preventive antioxidants. Vitamin E, glutathione, vitamin $\mathrm{C}$ and beta-carotene are some examples of the chain breaking type as they donate their own electron to the existing free radical and prevent the continuation of oxidation chain reaction. These antioxidants are found in the plasma and interstitial fluid $[28,29]$. The preventative category of antioxidants consists of enzymes that scavenge the free radicals before they initiate an oxidative attack.

Another way to categorize these antioxidants is based on their solubility in lipids or water. The antioxidants which are lipid soluble are located in the cellular membrane and lipoproteins, while the water soluble ones are located in the aqueous environments, such as the cytoplasm inside cells and the blood [30]. The following few sections discuss some of the important antioxidants of cells.

\subsection{Glutathione}

Glutathione (GSH) is one of the important cellular antioxidants. It is made up of three amino acids namely, cysteine, glutamic acid and glycine. All the cells of the body have the ability to synthesize glutathione, but it is found in high concentrations in the liver, lungs, and intestinal tract. The important functions of the glutathione include: anti-oxidation, detoxification, strengthening the immune system and signal transduction under stressful conditions. The diverse functions of GSH are due to the sulfhydryl group in cysteine, enabling it to chelate and detoxify harmful substances [31]. Additionally, glutathione also plays a role in processing medications and cancer-causing compounds (carcinogens), and in building DNA, proteins, and other important cellular components. Its usefulness as an antioxidative agent lies in its ability to donate electrons, and in that process it is oxidized to glutathione disulfide (GSSG) form. By donating an electron to a free radical, GSH can quench the unstable and highly reactive free radical and thus can prevent free radical mediated oxidative damage in the cells. GSSG is reduced to GSH by NADPH, an electron donor in this reaction. This reduction reaction is catalyzed by glutathione reductase. Thus the ratio between reduced and oxidized glutathione serves as an indicator of cellular toxicity mediated by oxidative damage. Large amounts of GSH are produced and stored in the liver, where it is used to detoxify harmful compounds. GSH levels are found to decline with age leaving the body susceptible to the damage by free radicals. 


\subsection{Vitamin $\mathrm{C}$, vitamin $\mathrm{E}$ and beta carotene}

Vitamin $C$ is a water soluble antioxidant which plays an important role in protecting the body against oxidative damage. Chemically, it is a carbohydrate-like substance. Human beings, unlike other mammals, do not have the ability to synthesize Vitamin C. Therefore, it must be furnished through the diet. Vitamin $C$ is easily oxidized and many functions in the body depend on this property. It stops the free radical chain reaction by donating an electron and prevents the reaction from progressing.

Carotenoids are a class of cellular antioxidants. Carotenoids are referred as provitamin A as they can potentially yield Vitamin A. Free radicals which can damage DNA and cause diseases like macular degeneration, coronary artery disease and cancer are neutralized by these carotenoids. Beta-carotene is perhaps the best known carotenoid. It was isolated in 1831 and its structure was determined in 1931.

Vitamin E is a fat soluble compound which also can impede oxidation to some degree [32]. Though there are eight forms in nature, only alpha-tocopherol form is functional in human beings. Vitamin E stops the production of ROS formation when fat undergoes oxidation. In addition to the above mentioned vitamins, essential trace elements, selenium, zinc, manganese, and copper, also play a critical role as antioxidants.

\subsection{Antioxidant enzymes}

Antioxidant enzymes inside the cells are the main defense against the free radicals. The most important antioxidant enzymes present inside the cells are superoxide dismutase (SOD), catalase, glutathione peroxidase (GPx), and glutathione S-transferase (GST). SOD catalyzes the dismutation of superoxide anion to $\mathrm{O}_{2}$ and $\mathrm{H}_{2} \mathrm{O}_{2}$. This reaction is considered to be the body's primary defense against free radicals as it prevents further generation of free radicals [29]. SOD is found in high levels in the liver, adrenal glands, kidney and spleen [33]. GPx, catalyzes the reduction of hydroperoxides and serves to protect the cell from hostile free radicals. Catalase, another antioxidant enzyme, along with GPx, is essential for removing hydrogen peroxide formed during oxidation reactions, and is found in high levels in liver, kidney and red blood cells. Both catalase and GPx enzymes exhibit a great degree of cooperativity in their action. Glutathione S-Transferase (GST) is yet another antioxidant enzyme primarily responsible for cellular defense mechanism against ROS. GST metabolizes xenobiotics, whether they are of endogenous or exogenous origin [34]. GST along with glutathione and GPx neutralizes free radicals and lipid hydroperoxides especially at low levels of oxidative stress [35]. The undesirable end products of lipid peroxidation are thus detoxified by GST. All of these enzymes prove to be indispensable in the cellular antioxidant defense mechanism and they are all necessary for the survival of the cell, even in normal conditions. An appropriate equilibrium between antioxidant enzyme activities is vital to ensure the cell survival during increased oxidative stress.

However some exogenous factors, such as environmental contaminants, have the potential to disrupt this equilibrium and cause incredible damage to the cells. The following section 
describes a specific group of brominated flame retardants called polybrominated diphenyl ethers (PBDEs).

\section{Polybrominated diphenyl ethers: A class of brominated flame retardants}

Fire accidents in the United States have the potential to cause severe economic problems for the individuals and for society at large. Each year, several thousand Americans die, several thousand more are injured, and millions of dollars of loss are all due to fires. To recover from these losses, individuals, their insurance companies, and society as a whole spend significant resources on temporary housing, groceries and meals, medical costs, psychological support, and relocation costs, to name a few expenditures. These ancillary costs may be 10-times higher than the cost to rebuild structures actually gutted by fire. To give a rough idea, the annual losses from natural calamities such as floods, hurricanes, tornadoes, earthquakes, etc., all put together in the United States equal just a fraction of total loss due to fires [36]. To comply with fire safety regulations in the United States manufacturers must follow strict guidelines in order to reduce the flammability of their products. For years, manufacturers have added chemicals to plastics and fabrics in order to reduce combustibility and increase overall safety of the products. Different types of brominated flame retardants have the promise of slowing down fire and hence are being extensively used. Figure 1 shows the chemical structures of four typical bromine compounds, namely, polybrominated biphenyls (PBBs), polybrominated diphenyl ethers (PBDEs), hexabromocyclododecane (HBCDs) and tetrabromobisphenol A (TBBPAs) [37].

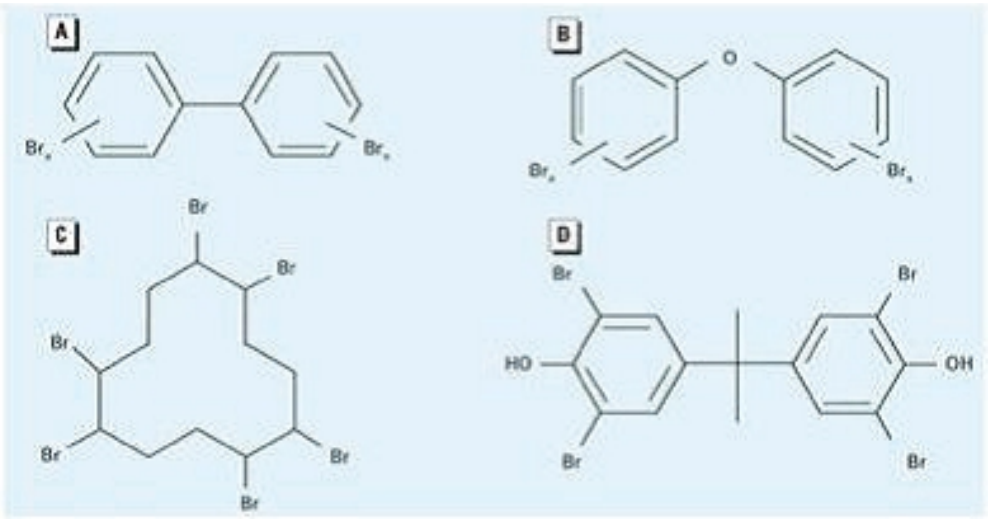

Figure 1. Chemical structures of BFRs. (A) PBBs, (B) PBDEs, (C) HBCD, and (D) TBBPA [37]

In the past few decades, use of PBDEs has become quite commonplace and these chemicals are added in large quantities to many commercial and household products, such as computers, TVs, foam mattresses, and carpets, to inhibit ignition and prevent fire accidents [37]. At high temperatures PBDEs liberate bromine atoms, which are effective at hindering 
the basic oxidizing reactions that drive combustion. Ever since PBDEs were introduced into the market, deaths due to fire accidents dropped significantly. Functionally, these flame retardants are similar to polychlorinated biphenyls (PCBs) that were banned from production in the U.S in the late 1970s due to potential adverse health effects and a persistent bio-accumulative nature [38]. Like PCBs, the PBDEs class contains 209 possible congeners. During the synthesis of PBDEs, a bromination reaction occurs on the biphenyl rings as one or more bromine atoms are attached to the biphenyl rings giving rise to one of the possible 209 possible congeners of PBDEs. The maximum number of bromine atoms that can attach to biphenyl rings is 10 and this brominated flame retardant is called decaBDE. Commercially these chemicals are available in three mixtures, pentaBDEs, octaBDEs and decaBDEs. Congeners with 1-5 bromine atoms are referred as lower BDEs and those with 6 10 are referred as higher BDEs.

\subsection{Production of PBDEs}

PBDEs were introduced into the market in the U.S. in the 1970s and peaked in the late 1990s. The production of various PBDEs in different regions of the world is shown in Table 1[37].

\begin{tabular}{|l|l|l|l|l|}
\hline BDE & Americas & Europe & Asia & Rest of the World \\
\hline Deca BDE & 24,500 & 7,600 & 23,000 & 1,050 \\
\hline Octa BDE & 1,500 & 610 & 1,500 & 180 \\
\hline Penta BDE & 7,100 & 150 & 150 & 100 \\
\hline Total BDEs & 33,100 & 8,360 & 24,650 & 1,330 \\
\hline
\end{tabular}

Data from BSEF (2001)

Table 1. BFRs produced (metric tons) in various regions of the world [37]

Table 2 shows the percent composition of various commercial PBDE mixtures. Currently, deca BDE constitutes $97 \%$ of the total production of PBDEs worldwide and it is the only BDE which is in use in the US [39].

\begin{tabular}{|l|l|l|l|l|l|l|l|}
\hline Product & Tetra & Penta & Hexa & Hepta & Octa & Nona & Deca \\
\hline Penta & $24-38$ & $50-60$ & $4-8$ & & & & \\
\hline Octa & & & $10-12$ & 44 & $31-35$ & $10-11$ & $<1$ \\
\hline Deca & & & & & & $<3$ & $<97$ \\
\hline
\end{tabular}

Table 2. Percent composition of commercial PBDE flame retardant mixtures [39].

Many research findings indicate that lower brominated congeners manifest greater affinity for lipids and accumulation. Hence they are more toxic and pose a greater health risk to humans and livestock than the higher brominated congeners [40]. 


\subsection{Exposure to PBDEs}

PBDEs are lipophilic and resistant to chemical and physical degradation. Due to their persistent nature, they have become widespread environmental contaminants and about 97\% of the American adult population has detectable levels of PBDEs [41]. Over the last 30 years the levels of these compounds in humans have increased and their levels in the US population are 10-100 times higher than levels measured in the Europe and Asia. According to a recent report, their levels in humans may be leveling off or decreasing due to their ban in many states of the US and European countries[39]. They are reported to be present in sediments, soil, outdoor and indoor air, household dust, foods, birds, fish, and terrestrial organisms [42].They have been detected in human serum, adipose tissue, and breast milk, and long-term exposure to these contaminants may pose a human health risk, especially to the developing fetus.

Despite their ban in many countries, vast amounts of these compounds are found to persist in existing consumer products, potentially contributing to environmental and human burdens for years to come. When released into the environment PBDEs adsorb rapidly onto solid particles such as soil or sediments due to their low water solubility and vapor pressure. Therefore, sediments and sewage sludge serve as a sink for these pollutants. These contaminants are still bioavailable even after adsorbing to the sediment particles [43]. PBDEs exposure to human being occurs through inhalation of household and workplace dust and by eating PBDE-contaminated foods. Exposure to PBDEs is nearly impossible to avoid as they are found in air, water and food. The fetus is also exposed to these compounds in utero, and after birth the newborn continues to be exposed from breast milk, where the toxins are transferred from the mother to the baby. A 25-year Swedish study found that the concentration of PBDEs in breast milk doubled every five years during the 25-year period [44]. The PBDE levels in breast milk from North American women are much higher than in breast milk from Swedish women, indicating greater exposure to PBDEs in this continent.

\subsection{Dietary intake of PBDEs}

The dietary intake of PBDEs from food in humans between ages 0-60 years are presented in Table 3. The intake of PBDEs was the highest in the first year during nursing. Breast milk has the highest levels of PBDEs. The intake of PBDEs per $\mathrm{kg}$ body weight reduced gradually with age. Children have higher PBDE intake than adults due to higher food intake per $\mathrm{kg}$ body weight [45].

\subsection{Adverse effects of PBDEs in laboratory animals}

Few toxic studies on PBDEs are reported in laboratory animals [46,47]. Some studies report these compounds to be toxic, carcinogenic and endocrine disruptors [48]. There are some reports indicating that PBDEs can induce disruption in spontaneous behavior, reduced habituation, impaired memory and learning in rats exposed during critical developmental period $[46,47]$. BDE-99 is a penta congener and it has been found to induce hyperactivity and 
impaired spermatogenesis in rats. Additionally, two recent in-vitro studies showed the induction of oxidative stress in rat neurons exposed to BDE-47 (another congener of pentaBDE congener) in primary cultured rat hippocampal neurons [49] and human cells [50] serving as note-worthy examples that call for more careful assessment of these toxic substances.

\begin{tabular}{|l|l|l|l|l|l|l|l|}
\hline Age in Years & $0-1$ & $2-5$ & $6-11$ & $12-19$ & $20-39$ & $40-59$ & $>60$ \\
\hline Diary & 0 & 427 & 293 & 372 & 250 & 203 & 175 \\
\hline Meat & 0 & 1,839 & 1,170 & 1,721 & 1530 & 1,408 & 1,094 \\
\hline Fish & 0 & 280 & 232 & 300 & 309 & 357 & 467 \\
\hline Eggs & 0 & 69 & 38 & 55 & 48 & 53 & 58 \\
\hline Margarine & 0 & 6 & 6 & 7 & 6 & 8 & 9 \\
\hline Butter & 0 & 30 & 17 & 19 & 22 & 15 & 22 \\
\hline Human Milk & 306,560 & 0 & 0 & 0 & 0 & 0 & 0 \\
\hline Total PBDE & & 2652 & 1755 & 2774 & 2164 & 2,084 & 1857 \\
\hline
\end{tabular}

Table 3. Daily intake of PBDEs from food (pg/kg body weight) [45]

\section{Lipid peroxidation during PBDE exposure}

Despite many studies, exact mechanism of toxicity of PBDEs is not yet clear and there is no definite explanation as to how PBDEs trigger the above mentioned deleterious effects. There appeared a school of thought that PBDEs, by reason of their electron affinity favor free radical formation in various tissues [51]. In the face of increased free radical generation the antioxidant enzymes namely, SOD, GPx, Catalase, and GST, are expected to give protection to the cells against the oxidative damage. An appropriate equilibrium between the antioxidant enzyme activities and free radicals is vital to ensure the cell's survival during increased oxidative stress. In the process of investigating the toxic mechanism authors have surmised oxidative stress induction in mice exposed to BDE-209, one of the most abundantly used flame retardant worldwide. This is based on a recent study showing the induction of oxidative stress in rat neurons exposed to BDE-47, a penta congener [49]. To interpret the adverse effects, both an assessment of the magnitude of free radical production and a careful evaluation of the threshold of protection in terms of antioxidant enzyme activity is necessary. Antioxidant enzymes such as SOD, GPx, GST, and catalase could also be the key indicators that could disclose the sequence of biochemical dysfunctions and alterations in signal transduction brought about by BDE-209. In the following few paragraphs the research protocols that one of the authors $(\mathrm{MCV})$ adopted to investigate the biological markers for oxidative stress following BDE-209 exposure are explicated. The study is performed on the liver and brain tissues of adult mice. The liver is a major biotransforming organ and brain is the most sensitive target to lipid soluble toxicants. Studying these two organs would give a clear picture of how this particular BDE is handled by these two vital organs. Further, since the metabolism and excretion of many PBDEs or for that matter any xenobiotics are tissue, species, sex and age dependent [52] it is intended to understand the effects of BDE-209 on these two vital organs; thus the hepato- and neurotoxic mechanisms could be simultaneously studied and correlated. 


\subsection{Materials and methods}

\subsubsection{Chemicals}

BDE- 85- CS (certified standard) was procured from Cambridge Isotope laboratories, Inc., Andover, MA, USA. Assay kits for the determination of lipid hydroperoxides, SOD, GPx, catalase, and GST were purchased from Cayman Chemical Company, Ann Arbor, Michigan, USA. BCA ${ }^{\mathrm{TM}}$ protein assay kit was purchased from Pierce Biotechnology Inc., Rockford IL, USA, for the protein assay of the sample. All other chemicals needed for the experiments were obtained from Sigma Aldrich Company.

\subsubsection{Animals}

Adult male Swiss Webster mice were obtained from Taconic Inc (Hudson, New York) and used for oxidative stress studies. Mice weighing $33 \pm 3 \mathrm{~g}$ were maintained at $23^{\circ} \mathrm{C} \pm 2^{\circ} \mathrm{C}$ with 12-hour light/12-hour dark photoperiods. Mice were provided food pellets and water ad libitum and were allowed to acclimatize for 15 days. The guidelines set forth by the Institutional Animal Care and Use Committee, Gannon University, Erie, Pennsylvania, were followed in the maintenance and use of mice. Mice were grouped into two groups randomly $(n=9)$. Group 1 was injected intraperitoneally with $0.25 \mathrm{mg} / \mathrm{kg}$ body weight of BDE-209 for four consecutive days and group 2 served as control and received same volume of vehicle solution, corn oil. Oral route of administration was not considered in this study as the aim of this work is to study the biochemical alterations induced at this particular concentration and administering via oral route results in elimination of some fraction of this chemical through feces. The present dosage of BDE-209 was selected based on some reports on other penta-BDE congeners in rodents [42,52]. In addition, the dosage used in the present study also is commensurate with the average levels of PBDEs (except BDE209) found in household dust [53]. On the fifth day mice were sacrificed and liver and brain tissues were separated, washed in phosphate-buffered saline (PBS), and weighed. For all the biochemical assays, a 5\% tissue homogenate of liver or brain was prepared using appropriate homogenizing buffers. The protocols that came with the assay kits were used for all biochemical assays and they are described below. The protein content of all the enzyme samples is assayed using Pierce ${ }^{\circledR}$ BCA Protein Assay Kit. Bovine serum albumin was used as standard.

\subsubsection{Lipid hydroperoxides}

Lipid peroxidation is quantified by a new method which directly quantifies the lipid hydroperoxides utilizing the redox reactions with ferrous ions.The liver and brain tissues from control and experimental mice were homogenized in nanopure water and the lipid hydroperoxides from the homogenate were extracted into chloroform. According to the protocol accompanying the lipid hydroperoxide assay kit, supplied by the Cayman Chemical Company, this chloroform extract was then used for the determination of lipid hydroperoxides. 


\subsubsection{Superoxide Dismutase (SOD)}

The liver and brain tissues from control and experimental mice were homogenized in 4-(2hydroxyethyl)-1-piperazineethanesulfonic acid (HEPES) buffer containing $1 \mathrm{mmole} / \mathrm{L}$ ethylene glycol tetraacetic acid (EGTA), $210 \mathrm{mmole} / \mathrm{L}$ mannitol and $70 \mathrm{mmole} / \mathrm{L}$ sucrose and centrifuged. The supernatant was used for the assay of SOD activity and was determined from the quantity of tetrazolium salt formed. One unit of SOD is defined as the amount of enzyme needed to exhibit $50 \%$ dismutation of superoxide anion.

\subsubsection{Glutathinoe Peroxidase (GPx)}

GPx activity was measured indirectly by a coupled reaction with glutathione reductase. Oxidized glutathione, produced upon reduction of hydroperoxide by GPx is recycled to its reduced state by glutathione reductase and NADPH. The oxidation of NADPH to $\mathrm{NADP}^{+}$is accompanied by a decrease in absorbance at $340 \mathrm{~nm}$. The rate of decrease in the $\mathrm{A}_{340}$ is directly proportional to the GPx activity in the sample. The tissues from control and exposed mice were homogenized in cold Tris- $\mathrm{HCl}$ with $5 \mathrm{mmole} / \mathrm{L}$ EDTA and $1 \mathrm{mmole} / \mathrm{L}$ dithiothreitol (DTT). The homogenate was centrifuged and the supernatant was used for the enzyme assay. The protocol described in GPx assay kit was followed.

\subsubsection{Catalase}

A tissue $5 \%$ homogenate of liver or brain was prepared in cold potassium phosphate buffer containing $1 \mathrm{mmole} / \mathrm{L}$ EDTA. The supernatant that surfaced was used for catalase assay as described in the assay protocol provided by Cayman Chemical Company. The catalase assay kit used the peroxidatic function of CAT for determination of enzyme activity. The enzyme reacts with methanol in the presence of an optimal concentration of $\mathrm{H}_{2} \mathrm{O}_{2}$. The formaldehyde produced is measured spectrophotometrically ( $=540 \mathrm{~nm}$ ) with 4-amino-3-hydrazino-5mercapto-1,2,4-triazole (Purpald) as the chromogen. Purpald specifically forms a bicyclic heterocycle with aldehydes, which upon oxidation changes from colorless to a purple color. One unit is defined as the amount of enzyme that will cause the formation of $1.0 \mathrm{nmol}$ of formaldehyde per minute at $250^{\circ} \mathrm{C}$.

\subsubsection{Glutathione S- transferase (GST)}

Tissues from control and experimental mice were homogenized in cold potassium phosphate containing $2 \mathrm{mmole} / \mathrm{L}$ EDTA. Homogenates were centrifuged and the supernatants used for the assay of Glutathione S-transferase, GST. GST activity was measured from the quantity of 1-Chloro-2, 4-Dinitrobenzene (CDNB) conjugated to glutathione. The one unit of enzyme conjugates 1.0 nmole of CDNB with reduced glutathione $/ \mathrm{min} / \mathrm{mg}$.

\subsubsection{Statistical treatment of the data}

Data presented in the Tables 4 and 5 are mean \pm standard deviation (SD) of 9 animals per group. The data were subjected to analysis of variance (ANOVA) and when means were 
significantly different they were further subjected to Tukey test. The percentage variation from controls was calculated and presented in the following results section. A value of $\mathrm{P}<$ 0.05 was considered significant.

\begin{tabular}{|l|l|l|l|}
\hline Oxidative Stress Marker & Control & BDE-209 & $\%$ change \\
\hline LPO & $2.06 \pm 0.63$ & $2.94 \pm 1.33$ & $+42.72^{*}$ \\
\hline SOD & $13.89 \pm 1.77$ & $19.25 \pm 2.56$ & $+38.58^{*}$ \\
\hline GPx & $103.43 \pm 14.77$ & $85.92 \pm 5.61$ & $-16.93^{*}$ \\
\hline Catalase & $58.78 \pm 14.22$ & $44.54 \pm 5.9$ & $-24.22 \cdot$ \\
\hline GST & $0.22 \pm 0.07$ & $0.17 \pm 0.05$ & $-22.73 \cdot$ \\
\hline
\end{tabular}

Table 4. The lipid hydroperoxides and antioxidant enzyme activities in the brain tissue. Values are mean \pm Standard deviation $(n=9)$. ${ }^{*}$ Significant at $p<0.05, \cdot$ not significant

\begin{tabular}{|l|l|l|l|}
\hline Oxidative Stress Marker & Control & BDE-209 & \% change \\
\hline LPO & $22.52 \pm 2.76$ & $27.31 \pm 1.98$ & $+21.67^{*}$ \\
\hline SOD & $33.37 \pm 1.59$ & $30.69 \pm 1.77$ & $-8.03^{*}$ \\
\hline GPx & $198.77 \pm 26.87$ & $143.78 \pm 7.8$ & $-28.01^{*}$ \\
\hline Catalase & $134.75 \pm 18.34$ & $92.78 \pm 10.54$ & $-31.15^{*}$ \\
\hline GST & $4.32 \pm 0.9$ & $2.97 \pm 0.3$ & $-31.3^{*}$ \\
\hline
\end{tabular}

Table 5. The lipid hydroperoxides and antioxidant enzyme activities in the liver tissue. Values are mean \pm Standard deviation $(n=9)$. ${ }^{*}$ Significant at $p<0.05$, -not significant

\section{Results}

The overall short term effect of BDE-209 over a period of four days is a remarkable disruption in the oxidant/antioxidant equilibrium. First, the levels of lipid hydroperoxides in the experimental tissues, liver and brain, have increased considerably. The percent increase in the levels of lipid hydroperoxides in liver was $27.67 \%(p<0.05)$ and in brain was $42.72 \%(p<0.05)$ over controls. These results are shown in Table 4 and 5 . The activities of SOD showed varied response in brain and liver. In liver, though there was a decrease in activity by $8.03 \%$, when compared to controls, it was not statistically significant. However in brain there was significant $((p<0.05)$ increase in activity $(38.58 \%)$. Another anti-oxidant enzyme activity measured was the GPx. The activity of this enzyme has been reduced in both brain $(16.93 \%)$ and liver tissues $(28.01 \%)$. These percentage changes also were significant at $p<0.05$ for both the tissues.

The enzyme catalase also suffered a similar reduction in activity over a four day exposure to BDE-209. Both in the brain and the liver tissues the catalase activity was lowered. In the brain there was a $24.22 \%$ of reduction and in liver it went down by $31.15 \%$. Just like GPx and catalase, GST also can reveal oxidative damage. In the present work GST activities were decreased in exposed liver by $31.3 \%(p<0.05)$ and in brain by about $22.73 \%(N S)$. All the above results clearly exhibited differential susceptibility of mice liver and brain tissues to BDE-209 - induced oxidative stress. 


\section{Discussion}

The results of this study clearly indicate the susceptibility of mice brain and liver tissues to BDE-209 induced damage when exposed to $0.25 \mathrm{mg} / \mathrm{kg}$ body weight for four consecutive days. The oxidative stress markers namely, lipid hydroperoxides, were found to increase in both the exposed liver and brain tissues. The increase in the lipid hydroperoxide levels could be due to an immediate perturbation in metabolizing enzymes in liver [54].The degree to which this can happen is yet open for further research. Liver being an important metabolizing organ hydrolyzes PBDEs in its attempt to eliminate them from the body. In this process the metabolites formed might possess greater toxicity than the parent BDE [55], thus making the liver prone to excessive damage. In the present study, BDE-209 exposed brains also showed significant increase in lipid hydroperoxides. Similar observations of increased peroxidation in the brain regions of adult rats exposed to two different concentrations of BDE-99 [42] lend support to this observation. In another study an increased peroxidation was noted in rat hippocampal neurons during BDE-47 exposure [49]. Additionally, there are reports on BDE-99 exposure in rats causing an increased ROS production in the tissues $[47,55]$. The mechanism responsible for ROS generation during PBDEs is not clear; however, it is suggested that PBDEs are electron acceptors under standard conditions prevailing in biological systems [51]. Reports on PBDE exposure and their involvement in the release of $[3 \mathrm{H}]$ arachidonic acid, protein kinase- $\mathrm{C}$ translocation, and disruption in calcium homeostasis [56,57] could further offer an explanation for the mechanism of ROS formation [49]. Thus the present observation of increased lipid hydroperoxide levels in the liver and brain tissues of mice substantiates higher lipid peroxidation process and oxidative stress following BDE-209 exposure.

The objective of the second part of this study was to examine how antioxidative enzymes responded to BDE-209 exposure in mice tissues. Although lipid hydroperoxides and lipid peroxidation have increased in the tissues, there was no corresponding increase in all the protective antioxidant enzymes studied, namely, SOD, GPx, catalase and GST in BDE-209 exposed mice tissues with the exception of brain SOD. The elevated lipid hydroperoxides under normal conditions should trigger an increase in antioxidant enzyme activity to suit the increased need to quench them. But in the present study there was a decrease in the activities of GPx, GST and catalse in the liver tissue. A decrease in catalase reflects an inability of the liver to remove hydrogen peroxide. As mentioned already, both catalase and GPx show high cooperativity in their action [58]. During the periods of increased hydroperoxide formation catalase activity depends on GPX activity to keep from being inactivated. On account of decreased GPx activity in BDE-209 exposed tissues of mice, the catalase might be inactivated by higher concentration of hydroperoxides. Glutathione-Stransferase is another antioxidant enzyme whose activity was reduced in liver tissue during BDE-209 exposure. BDE-99 exposure in rats showed a similar response of decreased antioxidant enzymes at $0.6 \mathrm{mg} / \mathrm{kg}$ bw [42]. In another report on BDE-99, rat liver and kidney showed an upsurge of lipid peroxidation and unstable antioxidant system [55], rendering support to our observations. The overall lowered activities of SOD, GPx and catalase in liver denote that liver tissue is not able to quench increased ROS and oxidative stress. These 
findings thus show that BDE- 209 brings about an oxidative stress in liver tissue not only through increased lipid hydroperoxide formation but also by reduced antioxidative enzyme protection.

The responses in the brain tissues were different with respect to SOD, catalase, and GST. Unlike in liver, SOD activity in brain was elevated indicating the presence of some degree of protection in the brain tissue against the oxidative damage. Though catalase and GST activities showed reduction (a trend as seen in the liver tissue) the values however, were not statistically significant. This variation in responses in brain compared to liver suggests that brain tissue was able to cope with increased lipid hydroperoxides to some degree, by way of increased SOD activity. The differences in responses in liver and brain tissues could be due to their intrinsic biochemical and functional differences. Brain with its high rate of $\mathrm{O} 2$ utilization, high levels of polyunsaturated lipids tends to respond differently [59]. A report on GST of rat brain regions after BDE-99 treatment showed similar response [47]. Further, the dosage used in this study could have resulted in this response in the brain tissue. A study [42] on the adult rat brains exposed to 2 different concentrations of BDE-99 showed contrasting results on TBARS (indicative of oxidative stress) levels. At lower dosage of exposure, the levels of TBARS were found to be reduced and at higher dosage they were increased. There are reports on rats showing decreased antioxidative enzymes in brain tissue during BDE-99 indicating that not all congeners have the same impact on the tissues. Another observation made by the authors (MCV) is BDE-209 is much less toxic compared to BDE-85 [60].

In summary, exposing mice to BDE-209 for four days has brought about an increased lipid hydroperoxide production as seen in the liver and brain tissues. The consequent peroxidation process has significantly impeded the activity of antioxidant enzymes, GPx, GST and catalase, in liver. Brain tissue on the other hand, showed elevated SOD, lowered GPx without significantly reducing catalase and GST activities. The results of this study indicate that BDE-209 congener of PBDEs has grave potential to disrupt the activity of antioxidant enzymes and induce oxidative stress. Additionally, liver seemed to be more susceptible to this toxicant than the brain. These differences in responses in these two tissues could be due to their inherent biochemical and functional differences.

\section{Conclusion}

Plasma membrane covers a cell and acts as a barrier between the internal and external environment thereof. It also regulates the movement of molecules across it. Chemically, the plasma membrane is a bi-layer composed of phospholipids and proteins. Membrane lipids form the bulk of the membrane itself and are more abundantly found than the proteins; however, they are smaller in size. Lipids serve as semipermeable barrier allowing only those materials that are lipid soluble and non-polar in nature to freely cross the plasma membrane. The membrane proteins give structural support and also have several functions including serving as transporters, receptors, enzymes and anchors. Carbohydrates which 
are attached to the membrane proteins serve as identification markers and help in the differentiation of self from non-self. Thus the plasma membrane is a very important structure of the cell and disruption in its structure leads to disruption in cellular function. The membrane lipids are prone to disruption and loss of selective permeability needed for normal metabolism especially through lipid peroxidation by free radicals.

Free radicals are atoms or molecules with one or more unpaired electrons in their outermost shell. Free radicals with oxygen are called reactive oxygen species (ROS). Examples of ROS include hydrogen peroxide, hydroxyl radicals, and superoxide anions. From time to time free radicals are generated as part of aerobic life during normal metabolism and in some instances these radicals are intentionally generated as part of normal signal transduction process. Superoxide anions are generated in cell organelles such as mitochondria and microsomes. These anions can react with biological molecules the most due to their longer half-life. Another most reactive free radical of the cell is hydroxyl radical. This radical is formed by the hemolytic fission of hydrogen peroxide. Free radicals, due to their high reactivity, attack other macromolecules within their vicinity and cause disruption of these molecules. When these free radicals attack proteins they change their biophysics thereby leading to functional disruption. They also earned notoriety in disrupting DNA and gene expression. It is reported that twenty types of alterations are introduced in the DNA by these ROS leading to variations in the gene expression. Last but not the least, ROS also can attack lipids especially the membrane lipids of the cell and cause extensive damage to the cellular function. Free radicals steal electrons from the polyunsaturated fatty acids (PUFAs) and other lipoproteins of the cells and initiate an attack called lipid peroxidation. The peroxidation of lipids occurs in three steps, namely, initiation, propagation and termination. In particular, lipid peroxidation characterized by low density lipoproteins plays an important role in atherosclerosis.

All cells have antioxidants in place to counteract the harmful effects of free radicals. Antioxidants are of two types, chain breaking antioxidants and preventive type. Vitamins such as $\mathrm{E}$ and $\mathrm{C}$ are examples for chain breaking type as they donate electrons to the existing free radicals and lessen their degree of reactivity to prevent further oxidative damage. The second category of antioxidants (the preventive type) consists of enzymes that scavenge the free radicals before they initiate oxidative attack. Glutathione is an important antioxidant of the cells whose function includes anti-oxidation, strengthening immune system, building DNA, and detoxification of medications and cancer causing compounds. The diverse functions of glutathione are mainly due to the sulfhydryl group in one of its amino acids known as cysteine. Vitamins C, E and provitamin A also play a role in protecting the body against the free radical induced damage. There are many enzymes inside the cells with antioxidant property. The most important antioxidant enzymes present inside the cells are superoxide dismutase (SOD), catalase, glutathione peroxidase, and glutathione Stransferase. SOD catalyzes the dismutation of superoxide anion to $\mathrm{O}_{2}$ and $\mathrm{H}_{2} \mathrm{O}_{2}$. GPx catalyzes the reduction of hydroperoxides and serves to protect the cell from the hostile free radicals. Catalase, another antioxidant enzyme, along with GPx, is essential for 
removing the hydrogen peroxide formed during oxidation reactions. GST along with glutathione and GPx neutralizes free radicals and lipid hydroperoxides especially at low levels of oxidative stress.

Polybrominated diphenyl ethers (PBDEs) are organic brominated flame retardants that were introduced into the market in 1970s and are added in large quantities to many commercial and household products such as computers, TVs, foam mattresses, carpets, etc., to inhibit ignition and prevent fire accidents. Ever since they were introduced into the market, deaths due to fire accidents abated significantly. Commercially these chemicals are available in three mixtures, pentaBDEs, octaBDEs and decaBDEs. Currently, deca BDE constitutes 97\% of the total production of PBDEs worldwide and it is the only BDE which is in use in the US. PBDEs are lipophilic and resistant to chemical and physical degradation. Due to their persistent nature, they have become widespread environmental contaminants. Avoiding exposure to PBDEs is nearly impossible as they are ubiquitously found in air, water and food. Few toxic studies on PBDEs are reported in laboratory animals. There are some reports indicating that PBDEs can induce disruption in spontaneous behavior, reduced habituation, impaired memory and learning in rats exposed during critical developmental period. When the toxic effect of BDE-209 was explored in the mice at $0.25 \mathrm{mg} / \mathrm{kg}$ body weight, it became clearly evident that this particular BDE triggers lipid hydroperoxide generation and increased lipid peroxidation process. Additionally, the antioxidant enzymes were diminished in liver, but in brain the levels of SOD increased without any change in catalase and GST. These results show that BDE-209 has the ability to elicit serious oxidative stress in mice with increased lipid peroxidation and decreased antioxidant protection. Research is underway to understand the effects of BDE-209 on the expression of genes and to find out if it are responsible for impairment of cell cycle regulation and causing apoptosis.

\section{Author details}

Mary C. Vagula* and Elisa M. Konieczko

Biology Department, Gannon University, Erie, PA, USA

\section{Acknowledgement}

The lead author (MCV) thanks Gannon University for granting the award of Faculty Research Grant. Authors also thank Prof. Gustafson and Dr. Masters for financial support and encouragement.

\section{References}

[1] Singer S, Nicholson G (1972) The fluid mosaic model of the structure of cell membranes. Science. 175(4023): 720-731.

\footnotetext{
* Corresponding Author
} 
[2] van Meer, G (2006) Cellular lipidomics. EMBO Journal. 24:3159-3165.

[3] Simons K, Sampaio J (2011) Membrane ogranization and lipid rafts. Cold Spring Harbor Perspectives in Biology. 3(10):a004697.

[4] Henneberry A, Wright M, McMaster C (2002) The major sites of cellular phospholipid synthesis and molecular determinants of fatty acid and lipid group specificity. Molecular and Cellular Biology. 13:3148-3161.

[5] Eisenberg D (1984) Three-dimensional structure of membrane and surface proteins. Annual Reviews in Biochemistry. 53:595-623.

[6] Driscoll P, Vuidepot A (1999) Peripheral membrane proteins. FYVE sticky fingers. Current Biology. 9:R857-R860.

[7] Gahmberg C, Tolvanen M (1996) Why mammalian cell surface proteins are glycoproteins. Trends in Biochemal Science 21:308-311.

[8] Commoner B, Townsend J, Pake G (1954) Free radicals in biological materials. Nature 174: 689-691.

[9] Tyler D (1975) Polarographic assay and intracellular distribution of superoxide dismutase in rat liver. Biochemistry Journal. 147:493-504.

[10] Ramos de Andrade Jr. D, Becco de Souza R, Alves dos Samtos S, Ramos de Andrade R (2005) Oxygen free radicals and pulmonary disease. Journal of Brazilian Pneumology. 31(1): 1-17.

[11] Gutteridge M, Halliwell B (1984) Oxygen toxicity, oxygen radicals, transition metals and disease. Biochemistry Journal. 219:1-14.

[12] Schibert J, Wilmer J (1991) Does hydrogen peroxide exist "free" in biological systems? Free radical biology. 11(6): 545-55.

[13] Oya-Ohta Y, Ochi T, Komoda Y, Yamamoto K (1995) The biological activity of hydrogen peroxide. VI. Mechanism of the enhancing effects of L-histidine: the role of the formation of a histidine-peroxide adduct and membrane transport. Mutation Research. 326(1):99-107

[14] Yu B (1994) Cellular defenses against damage from reactive oxidative species. Physiology Reviews. 74:139-62.

[15] Del Maestro R (1980) An approach to free radicals in medicine and biology. Acta Physiology Scandanavia. 492:153-68.

[16] Lennon S, Martin S, Cotter T (1991) Dose-dependent induction of apoptosis in human tumour cell lines by widely diverging stimuli. Cell Proliferation. 24 (2): 203-14.

[17] Griffiths H, Unsworth J, Blake D, Lunec J (1988) Free radicals in chemistry, pathology and medicine. London: Richelieu, pgs. 439-54.

[18] Marx G Chevion M (1986) Site-specific modification of albumin by free radicals. Reaction with copper (II) and ascorbate. Biochemistry Journal. 236:397-400.

[19] Wolff S, Dean S (1986) Fragmentation of protein by free radicals and its effect on their susceptibility to enzymic hydrolysis. Biochemistry Journal. 234:399-403.

[20] Morelli R, Russo-Volpe S, Bruno N, Lo Scalzo R (2003). Fenton-dependent damage to carbohydrates: free radical scavenging activity of some simple sugars. Journal of Agricultural and Food Chemistry 51(25): 7418-7425. 
[21] Sagone Jr. A, Greenwald E, Kraut J, Bianchine J, Singh D (1983) Glucose: role as free radical scavenger in biological systems. Journal of Laboratory and Clinical Medicine. 101:97-104.

[22] Wolff S, Crabbe M, Thornalley P (1984) The autoxidation of glyceraldehydes and other simple monosaccharides. Exp basel. 40: 244-6.

[23] Ames B (1983) Dietary carcinogens and anticarcinogens. Oxygen radicals and degenerative diseases. Science. 221:1256-64.

[24] Fraga C, Shigenaga M, Park, J Degan, P, Ames B (1990) Oxidative damage to DNA during aging: 8-hidroixy-deoxyguanosine in rat organ DNA and urine. Proceedings of the National Academy of Sciences USA. 87:4533-7.

[25] Dizdaroglu M, Jaruga P, Birincioglu M, Rodriguez H (2002) Free radical-induced damage to DNA: mechanisms and measurement. Free Radicals in Biology and Medicine. 32:1102-15.

[26] Cooke M, Evans M, Dizdaroglu M, Lunec J (2003) Oxidative DNA damage: mechanisms, mutation, and disease. FASEB Journal 17:1195-214.

[27] Kerr M, Bender C, Monti E (1996) An introduction to oxygen free radicals. Heart and Lung: the Journal of Acute and Critical Care. 25(3):200-208.

[28] McDermott J (2000) Antioxidant nutrients: current dietary recommendations and research update. Journal of American Pharmacology Association. 11(1):28-42.

[29] Scheibmeir H, Christensen K, Whitaker S, Jegaethesan J, Clancy R, Pierce J (2005) A review of free radicals and antioxidants for critical care nurses. Intensive and Critical Care Nursing. 21:24-28.

[30] Clark S (2002) The biochemistry of the antioxidants revisited. Nutrition in Clinical Practice. 17:5-17.

[31] Jozefczak M, Remans T, Vangronsveld J, Cuypers A (2012) Glutathione is a key player in metal-induced oxidative stress defenses. International Journal of Molecular Science. 13(3):3145-75.

[32] Traber M, Vitamin E. In: Shils M, Shike M, Ross A, Caballero B, Cousins R (eds.) Modern Nutrition in Health and Disease, $10^{\text {th }}$ ed. Baltimore, MD: Lippincott Williams Wilkins; 2006. P. 396-411.

[33] Halliwell B (1996) Antioxidants in human health and disease. Annual Review of Nutrition. 16:33-50.

[34] Sheweita S (1998) Heavy metal-induced changes in the glutathoine levels and glutathione reductase/glutathione-S-transferase activities in the liver of male mice. International Journal of Toxicology. 17:383-392.

[35] Yan H, Harding J (1997) Glycation induced inactivation and loss of antigenicity of catalase and superoxide dismutase. Biochemical Journal. 338:599-605.

[36] Fire in the United States, 2003-2007. Fifteenth edition, October 2009. FEMA. Executive summary. accessed at://www.usfa.fema.gov/downloads/pdf/statistics/fa_325.pdf

[37] Birnbaum, L, Staskal, D (2004) Brominated flame retardants: Cause for concern? Environmental Health Perspectives. 112: 9-17.

[38] Hites R (2004) Polybrominated diphenyl ethers in the environment and in people: A meta-analysis of concentrations. Environmental Science Technology. 38: 945-956. 
[39] Kimbrough K, Johnson W, Lauenstein G, Christensen J, Apeti D (2009) An Assessment of Polybrominated Diphenyl Ethers (PBDEs) in Sediments and Bivalves of the U.S. Coastal Zone. Silver Spring, MD. NOAA Technical Memorandum NOS NCCOS 94. 87.

[40] Gandhi N, Bhavsar S, Gewurtz S, Tomy G (2011) Can biotransformation of BDE-209 in lake trout cause bioaccumulation of more toxic, lower-brominated PBDEs (BDE-47, -99) over the long term? Environment International. 37(1):170-7.

[41] de Wit C (2002) An overview of brominated flame retardants in the environment. Chemosphere. 46 (5): 583-624.

[42] Belles M, Alonso V, Linares V (2010) Behavioral effects and oxidative status in brain regions of adult rats exposed to BDE-99. Toxicology Letters. 194(1-2): 1-7.

[43] Voorspoels S, Covaci A, Maervoet J, Schepens P (2004) PBDEs in marine and freshwater sediments from Belgium: levels, profiles and relations with biota. Journal of Environmental Monitoring. 6:914-918.

[44] Eriksson P, Jakobsson E, Fredriksson A (2004) Brominated Flame Retardants: A Novel Class of Developmental Neurotoxicants in our Environment? Environmental Health Perspectives 109(9): 903-908.

[45] Schecter A, Papke O, Harris T, Tung K, Musumba A, Olson J, Birnbaum L (2006) Polybrominated diphenyl ether (PBDE) levels in an expanded market basket survey of u.s. food and estimated pbde dietary intake by age and sex. Environmental Health Perspectives. 114(10) doi:10.1289/ehp.9121.

[46] Fischer C, Fredriksson A, Erilsson P (2008) Coexposure of neonatal mice to a flame retardant PBDE 99 (2,2'4,4'5-pentabromodiphenyl ether) and methyl mercury enhances developmental neurotoxic defects. Toxicology Science. 101(2):275-285.

[47] Cheng J, Gu J, Ma J, Chen X, Zhang M, Wang W (2009) Neurobehavioral effects, redox responses and tissue distribution in rat offspring developmental to BDE-99. Chemosphere 75: 963-968.

[48] McDonald T (2002) A perspective on the potential health risks of PBDEs. Chemosphere. 46: 745-755.

[49] He P, He W, Wang A, Xia T, Xu B, Zhang M, Chan X (2008) PBDE-47 induced oxidative stress, DNA damage and apoptosis in primary cultured rat hippocampal neurons. Neurotoxicology. 29(1):124-129.

[50] Kawashiro Y, Fukata H, Sato K, Aburatani H, Takigami H,Mori C (2009) Polybrominated diphenyl ethers cause oxidative stress in human umbilical vein endothelial cells. Human and Experimental Toxicology. 28(1):703-713.

[51] Zhao Y, Tao F, Zeng E (2008) Theoretical study on the chemical properties of polybrominated diphenyl ethers. Chemosphere. 70(5): 901-907.

[52] Chen L, Lebetkin E, Sanders J, Burka L (2006) Metabolism and disposition of 2,2',4,4',5 pentabromodiphenyl ether (BDE-99) following single or repeated administration to rats or mice. Xenobiotica. 36(6):515-534.

[53] Toms L, Hearn L, Kennedy K (2009) Concentrations of polybrominated diphenyl ethers in the matched samples of human milk, duct and indoor air. Environment International. 35(6):864-869. 
[54] Wolkers H, Boily F, Fink-Gremmels J, Bavel B, Hammill M, Primicerio R (2009) Tissue specific contaminant accumulation and associated effects on hepatic serum analytes and cytochrome p450 enzyme activity in hooded seals (cystophora cristata) from the gulf of St. Lawrence. Archives of Environmental Contamination. 56: 360-370.

[55] Albina M, Alonso V, Linares V (2010) Effects of exposure to (BDE-99) on oxidative status of liver and kidney in adult rats. Toxicology. 271(1-2): 51-56.

[56] Kodavanti P, Derr-Yellin E (2002) Differential effects of polybrominated diphenyl ethers and polychlorinated biphenyl on $[3 \mathrm{H}]$ arachidonic acid release in rat cerebellar granule neurons. Toxicological Sciences. 68(2):451-457.

[57] Kodavanti P, Ward T 2005. Differential effects of commercial polybrominated diphenyl and polychlorinated biphenyl mixtures on intracellular signaling in rat brain in vitro. Toxicological Sciences. 85(2):952-962.

[58] Baud O, Greene A, Li J, Wang H, Volpe J, Rosenberg P (2004) Glutathione peroxidasecatalase cooperativity is required for resistance to hydrogen peroxide by mature rat oligodendrocytes. The Jouranl of Neuroscience. 24(7):1531-1540.

[59] Sayre, L, Perry G, Smith M (2008) Oxidative stress and neurotoxicity. Chemical Research in Toxicology. 21, 172-188.

[60] Vagula M, Kubeldis N, Nelatury C (2011) Effects of BDE- 85 on the Oxidative Status and Nerve Conduction in Rodents. International journal of toxicology. 30(4): 428-434. 